\title{
Non-cooperative Support for the Asymmetric Nash Bargaining Solution
}

Citation for published version (APA):

Britz, V., Herings, P. J. J., \& Predtetchinski, A. (2010). Non-cooperative Support for the Asymmetric Nash Bargaining Solution. Journal of Economic Theory, 145(5), 1951-1967.

https://doi.org/10.1016/j.jet.2010.04.003

Document status and date:

Published: 01/09/2010

DOI:

10.1016/j.jet.2010.04.003

Document Version:

Publisher's PDF, also known as Version of record

Document license:

Taverne

Please check the document version of this publication:

- A submitted manuscript is the version of the article upon submission and before peer-review. There can be important differences between the submitted version and the official published version of record.

People interested in the research are advised to contact the author for the final version of the publication, or visit the DOI to the publisher's website.

- The final author version and the galley proof are versions of the publication after peer review.

- The final published version features the final layout of the paper including the volume, issue and page numbers.

Link to publication

\footnotetext{
General rights rights.

- You may freely distribute the URL identifying the publication in the public portal. please follow below link for the End User Agreement:

www.umlib.nl/taverne-license

Take down policy

If you believe that this document breaches copyright please contact us at:

repository@maastrichtuniversity.nl

providing details and we will investigate your claim.
}

Copyright and moral rights for the publications made accessible in the public portal are retained by the authors and/or other copyright owners and it is a condition of accessing publications that users recognise and abide by the legal requirements associated with these

- Users may download and print one copy of any publication from the public portal for the purpose of private study or research.

- You may not further distribute the material or use it for any profit-making activity or commercial gain

If the publication is distributed under the terms of Article $25 \mathrm{fa}$ of the Dutch Copyright Act, indicated by the "Taverne" license above, 


\title{
Non-cooperative support for the asymmetric Nash bargaining solution
}

\author{
Volker Britz $^{1}$, P. Jean-Jacques Herings ${ }^{*, 1}$, Arkadi Predtetchinski ${ }^{1}$ \\ Department of General Economics, Maastricht University, P.O. Box 616, 6200 MD Maastricht, The Netherlands \\ Received 11 November 2008; final version received 7 December 2009; accepted 27 December 2009
}

Available online 6 May 2010

\begin{abstract}
We study a model of non-cooperative multilateral unanimity bargaining on a full-dimensional payoff set. The probability distribution with which the proposing player is selected in each bargaining round follows an irreducible Markov process. If a proposal is rejected, negotiations break down with an exogenous probability and the next round starts with the complementary probability. As the risk of exogenous breakdown vanishes, stationary subgame perfect equilibrium payoffs converge to the weighted Nash bargaining solution with the stationary distribution of the Markov process as the weight vector.
\end{abstract}

(c) 2010 Elsevier Inc. All rights reserved.

JEL classification: $\mathrm{C} 78$

Keywords: Nash bargaining solution; Subgame perfect equilibrium; Stationary strategies; Markov process

\section{Introduction}

This paper contributes to the Nash program of supporting solution concepts from cooperative game theory by obtaining them as equilibrium outcomes of suitably constructed non-cooperative games. More specifically, we will be concerned with the asymmetric Nash bargaining solution. Consider a situation where two players receive a given pair of payoffs if they disagree, but may obtain any element of a convex set of other (superior) payoff pairs if they mutually agree on

\footnotetext{
* Corresponding author. Fax: +31 (0)43 3884878 .

E-mail addresses: v.britz@maastrichtuniversity.nl (V. Britz), p.herings@ maastrichtuniversity.nl (P.J.-J. Herings), a.predtetchinski@maastrichtuniversity.nl (A. Predtetchinski).

1 The authors would like to thank the Netherlands Organization for Scientific Research (NWO) for financial support.
} 
one such element. The Nash bargaining solution (NBS) is that payoff pair which maximizes the product of players' gains over their disagreement payoff. Nash [20] showed that this is the unique bargaining solution satisfying the axioms of scale invariance, symmetry, efficiency, and independence of irrelevant alternatives. One can generalize the NBS by assigning different weights to the players. The asymmetric Nash bargaining solution (ANBS) is that payoff pair which maximizes a weighted product of players' gains over their disagreement payoff, see Kalai [12].

The ANBS is used to gain insights on a wide variety of problems in economics. For instance, Bester [2] compares the effects of different pricing mechanisms on price and quality of a product. In particular, posted pricing is compared to bargaining between a buyer and a seller. In the latter case, the ANBS is taken to be the outcome of the bargaining interaction.

Another common application is wage bargaining between a firm and a union: Firm owners and workers can agree to produce and hence create a surplus. A part of the surplus goes to the workers as their wage, and the rest goes to the shareholders. If, however, the two sides cannot find an agreement, the workers may strike or the firm may shut down so that no surplus is generated. In a seminal paper on wage bargaining, Grout [7] studies the effect of different legal frameworks on wage bargaining. Throughout the analysis, it is assumed that bargaining between the firm and the union leads to the outcome predicted by the ANBS.

The prominent use of the ANBS in applications highlights the need for strong non-cooperative underpinnings of this concept. In the case of the ANBS, it is imperative to examine the noncooperative or strategic sources of players" "bargaining power" which is borne out in the weight vector of the ANBS. Nash [21] presents a non-cooperative demand game with two players who are uncertain about which payoff pairs are feasible. In the limit as the uncertainty vanishes, equilibrium payoffs converge to those predicted by the NBS. Carlsson [4] takes a similar approach, but with a different source of uncertainty: While the set of feasible payoffs is known to both players, their actions are subject to noise. If players make demands which do not exhaust the available surplus, the remainder is distributed according to an exogenously fixed rule. In the limit as the noise vanishes, there is a unique efficient equilibrium. The payoff pair is a particular ANBS; the bargaining weights are determined by the exogenous division rule.

In a seminal paper, Rubinstein [22] provides a non-cooperative game in which two players negotiate on the division of a pie. The players take turns acting as the proposer. The division of the pie in the unique subgame perfect equilibrium depends upon how strongly players prefer current over future payoffs. In the limit as players become perfectly patient, the equilibrium division converges to the NBS. In their discussion of cooperative and non-cooperative approaches to bargaining, Binmore, Rubinstein and Wolinsky [3] obtain the NBS in the limit if either players' impatience or the risk of an exogenous breakdown of the negotiations is vanishing.

Although the relationship between cooperative and non-cooperative approaches to bargaining is well understood for the case of two players, such is far less the case when more than two players are involved in the negotiation process. While it is straightforward to generalize the ANBS to $n$ players, the extension of its non-cooperative justification has turned out to be a much more difficult problem. Krishna and Serrano [14] make use of Lensberg's [17] stability (consistency) property. They design a non-cooperative bargaining protocol in which players can exit after partial agreements. This game has a unique subgame perfect equilibrium and the payoffs implied by that equilibrium converge to the NBS as the discount factor goes to one. Chae and Yang [5] obtain uniqueness of perfect equilibrium and convergence to the NBS in a game where a proposer negotiates with one responder at a time. In both papers, the results come at the cost of allowing partial agreements, rather than requiring unanimous consent to a comprehensive proposal. 
For the case with unanimous consent, an early support result for the NBS in the $n$ player case is implied by the analysis of Hart and Mas-Colell [9]. More recently, support results for the ANBS have been given by Miyakawa [19] and Laruelle and Valenciano [16]. All these papers consider a bargaining game where the proposer in each period is drawn from an invariant probability distribution. The stationary equilibrium payoffs turn out to converge to the ANBS with that probability distribution as the weight vector. Our analysis covers this result as a special case. Another special case is a fixed order of proposers, as analyzed by Kultti and Vartiainen [15], who also show that differentiability of the payoff set's Pareto frontier is essential for the convergence result if there are at least three players.

Other strands of the bargaining literature consider much more general bargaining protocols. For instance, in their analysis of uniqueness and efficiency of equilibria in bargaining games, Merlo and Wilson [18] assume that both the size of the cake to be divided and the order in which players propose and respond follow a Markov process. Kalandrakis [13] examines nodelay equilibria in stationary strategies under a Markov selection protocol, where agreement does not necessarily require unanimous consent of all players, but only of those within a winning coalition. The set of such equilibria is characterized and shown to be non-empty. Herings and Predtetchinski [10] study a game with Markov recognition probabilities, where the set of alternatives is one-dimensional. While studying delay or inefficiencies in bargaining games, other authors have used proposer protocols following stochastic processes, see for instance Cho and Duggan [6] and Hyndman and Ray [11]. In these papers, the stochastic process is not even required to have the Markov property, so the choice of the proposer may depend on aspects of history other than the identity of the previous proposer. Since more general selection protocols are used in much of the bargaining literature, we find it important to extend this approach to the support results for the ANBS.

In this paper we take a general approach towards multilateral bargaining. We aim at results for the case with $n$ players, a general set of feasible payoffs, and a general bargaining protocol. An informal description of the game we consider in this paper is as follows.

In the first period of an infinitely repeated bargaining game, the identity of the proposer is completely arbitrary. In each subsequent period, one out of the $n$ players is recognized as the proposer according to an irreducible Markov process. Upon recognition, the proposer offers a particular element of a convex and comprehensive set of feasible payoffs. If all players accept the offer, it is implemented. If a player rejects the offer, with some exogenously given and constant breakdown probability the game ends, whereas with the complementary probability the next period starts.

We show that subgame perfect equilibria in stationary strategies exist and we characterize the set of such equilibria. We then study the limit of an arbitrary sequence of such equilibria corresponding to a sequence of vanishing breakdown probabilities. We show that in the limit all players make the same proposal. Our main result is that in the limit this common proposal coincides with the ANBS with the stationary distribution of the Markov proposer selection process as the weight vector. Hence, equilibrium payoffs depend only on the set of feasible payoffs and the stationary distribution associated with the matrix of transition probabilities.

The proof of our result goes well beyond mere technical generalizations of existing proof strategies. Since the reservation payoff of a responding player depends on the identity of the current proposer, reservation payoffs cannot be expressed by a single vector, but correspond to a matrix. For any value of the exogenous breakdown probability, we consider the vectors corresponding to the difference between the equilibrium proposals of a pair of players. We show that in the limit as the breakdown probability vanishes, these vectors span an $(n-1)$-dimensional 
supporting hyperplane to the set of feasible payoffs at the point corresponding to the common limit proposal of the players. Finally, we demonstrate that the unique normal vector to this supporting hyperplane is proportional to the gradient of the asymmetric Nash product with weights equal to the stationary distribution associated with the matrix of transition probabilities.

Our analysis includes two very frequently encountered proposer protocols as special cases. First, consider the protocol where the proposer in each round is drawn from the same probability distribution. This corresponds to a Markov chain where all the rows of the transition matrix are the same. Our result implies that stationary equilibrium payoffs converge to the ANBS with the time-invariant probability distribution as bargaining weights. Consequently, our findings cover the support results of Hart and Mas-Colell [9], Miyakawa [19], and Laruelle and Valenciano [16]. Second, suppose that players make proposals in some fixed order, which is a straightforward $n$ player extension of the alternating-offer protocol in the classical paper by Rubinstein [22]. The fixed order of proposers induces a stationary distribution of the Markov chain with equal weights for all players. The limit equilibrium then corresponds to the NBS, the result shown by Kultti and Vartiainen [15]. In the current paper, we reveal how the aforementioned results are instances of a much more generally valid principle.

We assume that the set of feasible payoffs is comprehensive from below and that all weakly Pareto-efficient payoffs are also strongly Pareto-efficient, implying that the relevant bargaining space is $(n-1)$-dimensional. Herings and Predtetchinski [10] consider the same proposer selection protocol as in this paper, but study the case of a 1-dimensional set of alternatives where players have utility functions that are linear in the distance to their most preferred alternative. A unique prediction for the equilibrium payoffs is obtained in the limit as the discount factor goes to one. The equilibrium alternative of the bargaining game is contrasted with the prediction of both the median voter theorem and the ANBS and it is argued that there are no general relationships. Full dimensionality of the space of payoffs is therefore crucial to obtain convergence to ANBS.

The paper is organized as follows. Section 2 gives the formal description of the bargaining game and the definition of the equilibrium concept. In Section 3, we give necessary and sufficient conditions for a profile of stationary strategies to be an equilibrium in accordance with that concept. We also show that such an equilibrium exists. In Section 4, the main result is established: our non-cooperative support for the ANBS. Section 5 concludes.

\section{The bargaining game}

We consider the bargaining game $\Gamma(N, M, V)$. The set of players is denoted by $N$, and its members are indexed from 1 until $n$. The game is played for potentially infinitely many periods $t=0,1,2, \ldots$ In each period, one player acts as the proposer. In period $t=0$, the proposer is determined in an arbitrary way. In all later periods, the proposer is chosen by a Markov chain. The probability distribution on the players in period $t>0$ depends on the identity of the proposer in period $t-1$. The entry $m_{i j}$ of the matrix $M$ is the probability that player $j$ will propose in period $t$ given that player $i$ has proposed in period $t-1$. All entries of $M$ are non-negative and for each $i \in N$, it is true that $\sum_{j=1}^{n} m_{i j}=1$. The set $V$ corresponds to all feasible payoffs. We denote $V \cap \mathbb{R}_{+}^{n}$ by $V_{+}$. Our assumptions are as follows.

(A1) The set $V$ is closed, convex, and comprehensive from below. The origin lies in the interior of $V$. The set $V_{+}$is bounded and all weakly Pareto-efficient points in $V_{+}$are also strongly Pareto-efficient.

(A2) The matrix $M$ is irreducible. 
We denote the interior and boundary of a set $X$ by $\operatorname{int}(X)$ and $\partial X$ respectively. A vector $\eta$ with $\|\eta\|=1$ is said to be normal to the convex set $V$ at a point $\bar{v} \in V$ if $(v-\bar{v})^{\top} \eta \leqslant 0$ for every $v \in V$. The set of all vectors $\eta$ normal to $V$ at $\bar{v}$ is called the normal to $V$ at $\bar{v}$.

(A3) There is a unique vector in the normal to $V$ at every $v \in \partial V \cap \mathbb{R}_{+}^{n}$.

The assumption that all weakly Pareto-efficient points in $V_{+}$are also strongly Pareto-efficient is essential to our results. As we will show later in Section 3, this assumption implies that a proposal of a player $i$ gives all other players their respective reservation payoffs. Thus a proposer always extracts the full surplus from all other players. Our assumptions with respect to $V$ are similar to those in Merlo and Wilson [18] and Miyakawa [19].

The game proceeds as follows. In any period $t$, first the proposer is chosen in the aforementioned way. Next, the proposer offers a vector $v \in V$. Then, all players (including the proposer) decide sequentially whether to accept or reject the offer $v$, where for the sake of simplicity we assume that player $i$ responds before player $i+1$. We define the set $S(i)$ consisting of player $i$ and all its successors by $S(i)=\{j \in N \mid j \geqslant i\}$. If all players have accepted the vector $v$ in period $t$, the game ends and each player $i$ receives a payoff of $v_{i}$. As soon as one player rejects $v$, period $t+1$ starts with probability $\delta$, and the game ends with probability $1-\delta$. In the latter case, as well as in the case of perpetual disagreement, all players receive zero payoff. We assume that players maximize expected payoffs.

We denote by $H_{i}^{\mathrm{p}}$ the set of histories after which player $i$ has to make a proposal and by $H_{i}^{\mathrm{r}}$ the set of histories after which player $i$ has to respond to a proposal. Then, a strategy for player $i$ is a map $s_{i}: H_{i}^{\mathrm{p}} \cup H_{i}^{\mathrm{r}} \rightarrow V \cup\left\{\right.$ Yes, No\}, where $s_{i}\left(H_{i}^{\mathrm{p}}\right) \subset V$ and $s_{i}\left(H_{i}^{\mathrm{r}}\right) \subset\{$ Yes, No\}.

Player $i$ 's strategy is stationary if the same proposal is made at all histories $H_{i}^{\mathrm{p}}$ and if the action taken at any history $H_{i}^{\mathrm{r}}$ depends only on the current proposal and the current proposer. ${ }^{2}$

A Nash equilibrium is a profile of strategies from which no player has an incentive to unilaterally deviate. A subgame perfect Nash equilibrium (SPE) is a profile of strategies such that its restriction to any subgame is a Nash equilibrium of that subgame.

A stationary subgame perfect Nash equilibrium (SSPE) is a profile of stationary strategies which is an SPE.

\section{Analysis of stationary equilibrium}

In this section, we characterize the set of subgame perfect equilibria in stationary strategies. Theorem 3.11 gives the necessary and sufficient conditions for a strategy profile to be an SSPE and Theorem 3.12 asserts that an SSPE exists.

The analysis in this section resembles Kalandrakis [13], but some important differences should be noted: We conclude rather than assume that agreement is immediate in SSPE and we do not impose assumptions on the behavior of players who are indifferent between acceptance and rejection of some proposal. Furthermore, Banks and Duggan [1] have given an equilibrium

\footnotetext{
2 This notion of stationarity is weaker than the notion of subgame consistency due to Harsanyi and Selten [8], which implies that a player chooses the same action at any two nodes for which the continuation game is the same. For instance, suppose that rows $i$ and $j$ of $M$ are identical. Then, the continuation games after rejection of player $i$ 's proposal and that after rejection of player $j$ 's proposal are identical. Yet, our definition allows $A_{k}^{i}$ to be different from $A_{k}^{j}$ for one or more players $k \in N$.
} 
analysis similar to the one developed in this section, but not covering the general proposer protocol under consideration here.

Consider a profile of stationary strategies. It can be described by an $(n \times n)$-matrix $\Theta$, where the entry $\theta_{j}^{i}$ is the payoff proposed to player $j$ by player $i$, and a collection $\mathcal{A}$ of $n^{2}$ acceptance sets, where the acceptance set $A_{j}^{i}$ is the set of vectors in $V$ which player $j$ will accept when proposed by player $i$. The set of vectors in $V$ accepted by player $j$ and his successors, if proposed by $i$, is $A_{S(j)}^{i}=\bigcap_{k \in S(j)} A_{k}^{i}$. We refer to $A^{i}=A_{S(1)}^{i}=\bigcap_{j \in N} A_{j}^{i}$ as the social acceptance set for proposer $i$.

Suppose that in period $t$, the proposal of player $i$ is rejected. With probability $1-\delta$ the game ends and all players receive zero payoff, and with probability $\delta$ period $t+1$ is reached and play proceeds according to the profile $(\Theta, \mathcal{A})$ of stationary strategies. The expected payoff to player $j$ after rejection is $r_{j}^{i}(\Theta, \mathcal{A})$. Omitting the argument $(\Theta, \mathcal{A})$ from the notation wherever possible, we refer to $r_{j}^{i}$ as the reservation payoff of player $j$ when $i$ proposes.

Proposition 3.1. The reservation payoff $r^{i}$ belongs to int $(V)$.

Proof. Conditional on the next period being reached, the payoffs are determined by a probability distribution on $V$ (notice that also $0 \in V$ ), so expected payoffs belong to $V$ since $V$ is convex. Since with probability $1-\delta$ the next period is not reached, these expected payoffs equal $\delta^{-1} r^{i}$, so $\delta^{-1} r^{i} \in V$. Since $0 \in \operatorname{int}(V)$, the convex combination $(1-\delta) 0+\delta \delta^{-1} r^{i}=r^{i}$ belongs to $\operatorname{int}(V)$.

One implication of Proposition 3.1 is that a proposer always has the option to make a proposal that strictly exceeds the reservation payoff of every player.

Proposition 3.2. In SSPE, for $j \in N$, if $v \in A_{S(j)}^{i}$, then $v_{k} \geqslant r_{k}^{i}$ for all $k \in S(j)$.

Proof. Suppose that $(\Theta, \mathcal{A})$ is a profile of stationary strategies such that $v \in A_{S(j)}^{i}$ but $v_{k}<r_{k}^{i}$ for some player $k \in S(j)$. Consider a history in $H_{k}^{\mathrm{r}}$, where player $k$ responds to the proposal $v$ made by player $i$. At that history player $k$ could deviate from $(\Theta, \mathcal{A})$ by rejecting $v$. In that case, an expected payoff of $r_{k}^{i}$ would result. Hence, this deviation is profitable and $(\Theta, \mathcal{A})$ cannot be an SSPE.

Proposition 3.2 implies that for a vector of payoffs $v$ to belong to the social acceptance set, it should satisfy $v_{j} \geqslant r_{j}^{i}$ for all $j \in N$.

Proposition 3.3. In SSPE (a) if $v \in V$ is such that $v_{n}>r_{n}^{i}$, then $v \in A_{n}^{i}$, and (b) for each $j=$ $1, \ldots, n-1$ if $v \in A_{S(j+1)}^{i}$ is such that $v_{j}>r_{j}^{i}$, then $v \in A_{j}^{i}$.

Proof. To prove part (a) of the proposition suppose that $v \in V$ satisfies $v_{n}>r_{n}^{i}$. Consider a history where player $n$ has to respond to the proposal $v$ of player $i$. If $n$ accepts, the proposal will be implemented, so a payoff of $v_{n}$ will result, which is strictly greater than the payoff $r_{n}^{i}$ which would result from a rejection. Therefore, SSPE requires that $v \in A_{n}^{i}$.

To prove part (b) take a $j=1, \ldots, n-1$ and a $v \in A_{S(j+1)}^{i}$ such that $v_{j}>r_{j}^{i}$. Consider a history where player $j$ responds to the proposal $v$ of player $i$. If player $j$ accepts, the proposal 
will be implemented since all the players succeeding $j$ accept. Since rejection results in a payoff of $r_{j}^{i}$, SSPE requires that $v \in A_{j}^{i}$.

Proposition 3.3 established a kind of converse of Proposition 3.2. One implication of Proposition 3.3 is that a vector $v \in V$ that satisfies $v_{j}>r_{j}^{i}$ for all $j \in N$ belongs to the social acceptance set $A^{i}$.

Proposition 3.4. In SSPE, each player's proposal $\theta^{i}$ lies in the social acceptance set $A^{i}$ for proposer $i$.

Proof. Suppose by way of contradiction that under some SSPE there is a player $i \in N$ such that $\theta^{i} \notin A^{i}$. Consider the subgame starting at a history where player $i$ is the proposer. Since $\theta^{i}$ is rejected, $r^{i}$ is the vector of expected payoffs by definition. By Proposition 3.1, $r^{i} \in \operatorname{int}(V)$. Consequently, there exists $v \in V$ such that $v_{j}>r_{j}^{i}$ for all $j \in N$. By the previous proposition, $v \in A^{i}$. Hence, it would be a profitable deviation for player $i$ to propose $v$ instead of $\theta^{i}$.

Proposition 3.5. In SSPE, $\theta_{j}^{i} \geqslant 0$ and $r_{j}^{i} \geqslant 0$ for all $(i, j) \in N \times N$.

Proof. Suppose by way of contradiction that $(\Theta, \mathcal{A})$ is an SSPE and that $\theta_{j}^{i}<0$ for some $(i, j) \in$ $N \times N$. Consider a history where player $j$ has to respond to the proposal $\theta^{i}$. By Proposition 3.4, $\theta^{i} \in A^{i}$, so player $j$ will receive a strictly negative payoff if play proceeds according to $(\Theta, \mathcal{A})$. But then, it would be a profitable deviation for player $j$ to reject the proposal. Consequently, it holds that $\theta_{j}^{i} \geqslant 0$ for all $(i, j) \in N \times N$. It then follows that $r_{j}^{i} \geqslant 0$ for all $(i, j) \in N \times N$.

The next proposition shows that an equilibrium proposal of any player gives all other players their respective reservation payoffs. Thus a proposer always extracts the entire surplus from the other players.

Proposition 3.6. In SSPE, $\theta_{j}^{i}=r_{j}^{i}$ for all $(i, j) \in N \times N$ such that $i \neq j$.

Proof. Since $\theta^{i} \in A^{i}$ by Proposition 3.4, Proposition 3.2 implies that $\theta_{j}^{i} \geqslant r_{j}^{i}$ for all $j \in N$. Suppose $\theta_{k}^{i}>r_{k}^{i}$ for some $k \in N$ such that $k \neq i$. Define the vector $v$ as follows,

$$
v_{j}= \begin{cases}\theta_{i}^{i} & \text { if } j=i \\ r_{j}^{i} & \text { if } j \neq i\end{cases}
$$

The vector $v$ is clearly non-negative and it is in $V$, because $v \leqslant \theta^{i}$ and $V$ is comprehensive. Furthermore, $v_{k}=r_{k}^{i}<\theta_{k}^{i}$, so the vector $v$ is dominated by $\theta^{i}$ and is therefore not strongly Pareto-efficient. Since we assume that all weakly Pareto-efficient vectors of $V_{+}$are also strongly Pareto-efficient, the vector $v$ is not weakly Pareto-efficient. Thus, there exists a vector $v^{\prime}$ such that $v_{j}^{\prime}>v_{j}$ for all $j \in N$.

We show now that $v^{\prime} \in A^{i}$. Indeed, $v_{j}^{\prime}>v_{j}=r_{j}^{i}$ for all $j \neq i$. And for player $i$ we have the inequality $v_{i}^{\prime}>v_{i}=\theta_{i}^{i} \geqslant r_{i}^{i}$. Thus we conclude that $v_{j}^{\prime}>r_{j}^{i}$ for all $j \in N$. Proposition 3.3 now implies that $v^{\prime} \in A^{i}$, as desired. 
But then player $i$ has a profitable deviation at any history where he is entitled to make a proposal, namely propose the vector $v^{\prime}$ rather than $\theta^{i}$. Indeed, the vector $v^{\prime}$ is accepted and results in a payoff of $v_{i}^{\prime}>\theta_{i}^{i}$ to player $i$.

Proposition 3.7 claims that all players make a proposal belonging to the boundary of $V$.

Proposition 3.7. In SSPE, $\theta^{i} \in \partial V$ for each $i \in N$.

Proof. Suppose by way of contradiction that there is a player $i \in N$ such that $\theta^{i} \in \operatorname{int}(V)$. Equivalently, there exists $v \in V$ such that $v_{j}>\theta_{j}^{i}$ for all $j \in N$. By the immediate agreement property, $\theta^{i} \in A^{i}$. This implies that $v \in A^{i}$ as well. But then it would be a profitable deviation for player $i$ to propose $v$ rather than $\theta^{i}$.

The previous propositions are collected in the following theorem.

Theorem 3.8. Suppose $(\Theta, \mathcal{A})$ is an SSPE profile inducing reservation payoffs $r^{i}$. For each $i \in N$

$$
\begin{aligned}
& \theta^{i} \in A^{i} \cap \partial V \cap \mathbb{R}_{+}^{n}, \\
& r^{i}=\delta \sum_{k=1}^{n} m_{i k} \theta^{k}, \\
& \theta_{j}^{i}=r_{j}^{i}, \quad j \in N \backslash\{i\}, \\
& A_{S(j)}^{i} \subset \bigcap_{k \in\{j, \ldots, n\}}\left\{v \in V \mid v_{k} \geqslant r_{k}^{i}\right\}, \quad j \in N, \\
& A_{n}^{i} \supset\left\{v \in V \mid v_{n}>r_{n}^{i}\right\}, \\
& A_{j}^{i} \supset\left\{v \in A_{S(j+1)}^{i} \mid v_{j}>r_{j}^{i}\right\}, \quad j \in\{1, \ldots, n-1\} .
\end{aligned}
$$

In what follows, we establish the converse; the conditions of Theorem 3.8 characterize the set of SSPE. To do so, we will first state the well-known one-shot deviation principle.

Proposition 3.9. Let $(\Theta, \mathcal{A})$ be a stationary strategy profile satisfying (3.1)-(3.6). If there is a subgame such that some player has a profitable deviation, then there is a subgame where he has a profitable one-shot deviation.

Here, a one-shot deviation in a subgame is a single deviation by the player at the root of the subgame. The proof of this principle is standard in the literature and is based on the optimality principle from dynamic programming. We will show next that no player has a profitable one-shot deviation from a profile of strategies satisfying conditions (3.1)-(3.6).

Proposition 3.10. Let $(\Theta, \mathcal{A})$ be a stationary strategy profile satisfying (3.1)-(3.6). There is no subgame where a player has a profitable one-shot deviation.

Proof. Consider the subgame at a history $h \in H_{i}^{\mathrm{p}}$. Suppose player $i$ has a one-shot deviation involving a proposal $v^{i}$ different from $\theta^{i}$. If $v^{i}$ does not belong to $A^{i}$, it leads to a payoff $r_{i}^{i}$ for player $i$. Since $\theta_{j}^{i}=r_{j}^{i}$ for all $j \neq i$ and $r^{i} \in \operatorname{int}(V)$, the Pareto-efficiency of $\theta^{i}$ implies $\theta_{i}^{i}>r_{i}^{i}$, 
so the deviation is not profitable. If $v^{i}$ belongs to $A^{i}$, then, for $j \neq i, v_{j}^{i} \geqslant r_{j}^{i}=\theta_{j}^{i}$. Now $v_{i}^{i} \leqslant \theta_{i}^{i}$, since otherwise the Pareto-efficiency of $\theta^{i}$ would be violated. The deviation is not profitable.

Consider a subgame at a history $h \in H_{j}^{\mathrm{r}}$ where player $j$ has to respond to a proposal $v$ made by player $i$. If $j \in\{1, \ldots, n-1\}$ and $v \notin A_{S(j+1)}^{i}$, then either action by player $j$ leads to a payoff of $r_{j}^{i}$ and hence the deviation is not profitable. Suppose now that either $j=n$ or $j \in\{1, \ldots, n-1\}$ and $v \in A_{S(j+1)}^{i}$. If $v \in A_{j}^{i}$ then the original strategy leads to a payoff of $v_{j}$ while the deviation yields a payoff of $r_{j}^{i}$. Since $v_{j} \geqslant r_{j}^{i}$ by condition (3.4), the deviation is not profitable. If $v \notin A_{j}^{i}$ then the original strategy leads to a payoff of $r_{j}^{i}$ while the deviation yields a payoff of $v_{j}$. Since $v_{j} \leqslant r_{j}^{i}$ by conditions (3.5)-(3.6), the deviation is not profitable.

The previous two propositions imply that the strategies conforming to the conditions of Theorem 3.8 are subgame perfect. Since they are also stationary, we have the following.

Theorem 3.11. The strategy profile $(\Theta, \mathcal{A})$ fulfills conditions (3.1)-(3.6) if and only if it is an SSPE.

If a matrix $\Theta$ of proposals is part of an SSPE, then it is part of many SSPE's. This inessential multiplicity has two sources. First, if a responding player is proposed exactly the reservation payoff, then our characterization restricts behavior only if the proposal on the table is the equilibrium proposal. This is reflected by the fact that each point on the boundary of the social acceptance set (except the relevant equilibrium proposal) may or may not be an element of that set in SSPE. Second, if a proposal lies outside a social acceptance set, it is indeterminate which player will reject the proposal. Consider for example the case where $N=\{1,2,3,4\}$ and $r^{1}=(1,1,1,1)$. Now suppose that player 1 has proposed $v=(2,2,0,0)$ in some subgame. Since $v_{3}<r_{3}^{1}$ and $v_{4}<r_{4}^{1}$, Proposition 3.2 implies that $v \notin A^{1}$, and by Proposition $3.4 v \neq \theta^{1}$. It is also true that $v \notin A_{4}^{1}$ : If the node where player 4 has to respond is reached, that player effectively chooses between a payoff of 1 and a payoff of 0 , so SSPE requires rejection of the proposal. However, the SSPE characterization leaves indeterminate whether players 1,2 , and 3 will accept or reject $v$. Consequently, there is an SSPE for any configuration of responses by these players. In particular, player 3 may accept $v$ in SSPE although $v_{3}<r_{3}^{1}$, and player 2 may reject $v$ although $v_{2}>r_{2}^{1}$. This reasoning even extends to player 1: In SSPE, it is possible that $v \notin A_{1}^{1}$ although $v_{1}>r_{1}^{1}$ and player 1 is the proposer. However, this does not mean that player 1 may reject his own proposal on the equilibrium path, since the SSPE characterization requires the specific proposal $\theta^{1}$ to be made and immediately accepted by all players.

Theorem 3.12. An SSPE exists.

For a proof of SSPE existence, we refer to Kalandrakis [13].

\section{The limit equilibrium}

Our proofs so far did not rely on Assumptions (A2) and (A3). They will be needed for the results of this section. Since the matrix $M$ is irreducible, it has a unique stationary distribution denoted by $\mu$. Recall that the stationary distribution $\mu$ is a probability distribution on the set of players $N$ satisfying the equation $\mu M=\mu$. Furthermore, irreducibility of $M$ implies that all 
states occur with positive probability under the stationary distribution, that is $\mu_{i}>0$ for each $i \in N$. If the matrix $M$ was reducible, the state space of $M$ could be partitioned into several communicating classes. In this case, one obtains results analogous to those in the sequel within each communicating class.

Theorem 4.2 below is the main result of the paper. As the continuation probability goes to one, along any sequence of stationary subgame perfect equilibria of $\Gamma$, the equilibrium proposal of all players converges to the same limit. This common limit is the asymmetric Nash bargaining solution weighted by the stationary distribution $\mu$, denoted $\mu$-ANBS.

Definition 4.1. The asymmetric Nash product with weights $\mu$ is the function $\rho: V_{+} \rightarrow \mathbb{R}$ defined by

$$
\rho(v)=\prod_{i \in N}\left(v_{i}\right)^{\mu_{i}} .
$$

The $\mu$-ANBS is the unique maximizer of the function $\rho$ on the set $V_{+}$.

Theorem 4.2. Let $\left\{\delta_{h}\right\}_{h \in \mathbb{N}}$ be a sequence of continuation probabilities in $[0,1)$ converging to 1 . For each $h$, let $\Theta\left(\delta_{h}\right)$ be a matrix of proposals in some SSPE of the game $\Gamma$ with continuation probability $\delta_{h}$. Then the limits $\lim _{h \rightarrow \infty} \theta^{i}\left(\delta_{h}\right)$ exist for each $i \in N$. All limits are equal to the $\mu-A N B S$.

Let $\delta_{h}$ and $\Theta\left(\delta_{h}\right)$ be as in Theorem 4.2. The sequence $\left\{\Theta\left(\delta_{h}\right)\right\}$ has a convergent subsequence, as it lies in the compact set $V_{+}^{n}$, the Cartesian product of $n$ copies of $V_{+}$. For the remainder of this section, we will fix any such convergent subsequence and denote its limit by $\bar{\Theta}$. Since the convergent subsequence considered is arbitrary, to prove Theorem 4.2 it is sufficient to show that each column of the matrix $\bar{\Theta}$ is the $\mu$-ANBS.

We now give a brief overview of the argument. First we show that along the sequence $\left\{\Theta\left(\delta_{h}\right)\right\}$ of equilibria the proposals of all players converge to a common limit, say the point $\bar{\theta} \in V$. We then compute the tangent space to the set $\partial V$ at the point $\bar{\theta}$ by considering the pairwise differences of the equilibrium proposals of players $i$ and $n$, and show they converge to zero at the same speed as $1-\delta_{h}$. In fact, we are able to compute the limits of the vectors $\left(\theta^{i}\left(\delta_{h}\right)-\theta^{n}\left(\delta_{h}\right)\right) /\left(1-\delta_{h}\right)$ explicitly, which are then shown to span the tangent space to the set $\partial V$ at the point $\bar{\theta}$. Using this result we show next that the tangent space at $\bar{\theta}$ is orthogonal to the gradient of the asymmetric Nash product with weights $\mu$, thereby showing that $\bar{\theta}$ is the $\mu$-ANBS.

\section{Proposition 4.3. All columns of $\bar{\Theta}$ are identical.}

Proof. For any $i \neq j$, it follows from the SSPE characterization that

$$
\bar{\theta}_{j}^{i}=\sum_{k=1}^{n} m_{i k} \bar{\theta}_{j}^{k} .
$$

Suppose that, contrary to the proposition, not all limit proposals $\bar{\theta}^{1}, \ldots, \bar{\theta}^{n}$ are the same. Let $j \in N$ be such that in the limit not all players propose the same to player $j$, and choose $\bar{\theta}_{j}^{\diamond}$ to be either $\min _{i \in N}\left\{\bar{\theta}_{j}^{i}\right\}$ or $\max _{i \in N}\left\{\bar{\theta}_{j}^{i}\right\}$, whichever is not equal to $\bar{\theta}_{j}^{j}$. Define $N^{\diamond}=\left\{i \in N \mid \bar{\theta}_{j}^{i}=\bar{\theta}_{j}^{\diamond}\right\}$. For any $i \in N^{\diamond}$, we have 


$$
\bar{\theta}_{j}^{\diamond}=\bar{\theta}_{j}^{\diamond} \sum_{k \in N^{\diamond}} m_{i k}+\sum_{k \in N \backslash N^{\diamond}} m_{i k} \bar{\theta}_{j}^{k},
$$

which is equivalent to

$$
\bar{\theta}_{j}^{\diamond} \sum_{k \in N \backslash N^{\diamond}} m_{i k}=\sum_{k \in N \backslash N^{\diamond}} m_{i k} \bar{\theta}_{j}^{k} .
$$

Suppose first that for some $i \in N^{\diamond}$,

$$
\sum_{k \in N \backslash N^{\diamond}} m_{i k}>0 .
$$

Then

$$
\bar{\theta}_{j}^{\diamond}=\frac{\sum_{k \in N \backslash N^{\diamond}} m_{i k} \bar{\theta}_{j}^{k}}{\sum_{k \in N \backslash N^{\diamond}} m_{i k}},
$$

which contradicts the fact that either $\bar{\theta}_{j}^{\diamond}<\bar{\theta}_{j}^{k}$ for all $k \in N \backslash N^{\diamond}$ or $\bar{\theta}_{j}^{\diamond}>\bar{\theta}_{j}^{k}$ for all $k \in N \backslash N^{\diamond}$. Therefore, $m_{i k}=0$ for all $(i, k) \in N^{\diamond} \times\left(N \backslash N^{\diamond}\right)$ and thus $N^{\diamond}$ is an absorbing set. Since $N^{\diamond} \neq N$, this contradicts the irreducibility assumption on $M$.

We denote a column of $\bar{\Theta}$ by $\bar{\theta}$. For $i \in N \backslash\{n\}$, we define

$$
d^{i}\left(\delta_{h}\right)=\frac{1}{1-\delta_{h}}\left(\theta^{i}\left(\delta_{h}\right)-\theta^{n}\left(\delta_{h}\right)\right) .
$$

Let $D\left(\delta_{h}\right)$ be the $(n \times(n-1))$-matrix with columns $d^{1}\left(\delta_{h}\right), \ldots, d^{n-1}\left(\delta_{h}\right)$.

The rest of the proof is organized as follows. In Proposition 4.5 we compute the limits of $d^{i}\left(\delta_{h}\right)$ as $h$ goes to infinity. Proposition 4.6 establishes that the limit of $\left\{d^{i}\left(\delta_{h}\right)\right\}$ belongs to the tangent space to $\partial V$ at $\bar{\theta}$. We then proceed to show in Proposition 4.8 that the limits of $d^{1}\left(\delta_{h}\right), \ldots, d^{n-1}\left(\delta_{h}\right)$ are linearly independent and thus span the tangent space to $\partial V$ at $\bar{\theta}$. And finally, Proposition 4.9 establishes that the gradient of the Nash product with weights $\mu$ at the point $\bar{\theta}$ is orthogonal to the tangent space of $V$, thereby showing that $\bar{\theta}$ is the $\mu$-ANBS.

For a matrix $A$, we denote by $A_{-i}$ and $A^{-i}$ the matrix $A$ without its $i$ th row and column, respectively. We write $\mathbb{1}$ for a column vector of ones and $I$ for the identity matrix.

For $j \in N$, we define the matrix $L(j)$ by

$$
L(j)=\left[M^{\top}-I\right]_{-n}^{-j} .
$$

Thus $L(j)$ is the $((n-1) \times(n-1))$-matrix obtained from $M^{\top}-I$ by deleting column $j$ and row $n$. Proposition 4.4 is an auxiliary result used in the proof of Proposition 4.5.

Proposition 4.4. The matrix $L(j)$ is invertible for all $j \in N$.

Proof. Suppose $L(j)$ is singular. Let $a$ be a non-zero vector such that $\left[M^{\top}-I\right]_{-n}^{-j} a=0$. Since the elements in any column of the matrix $M^{\top}-I$ add up to zero, we also have the equation $\left(M^{\top}-I\right)_{n}^{-j} a=0$, so $\left[M^{\top}-I\right]^{-j} a=0$. By using $\left[M^{\top}-I\right]^{-j}=\left(M^{\top}-I\right) I^{-j}$ and defining $b=I^{-j} a$, we see that $\left(M^{\top}-I\right) b=0$. Thus the vector $b$ is an eigenvector of $M^{\top}$ associated with eigenvalue 1. By the Perron-Frobenius theorem, any non-zero eigenvector of $M^{\top}$ associated with eigenvalue 1 is a strictly positive vector. However, since $b_{j}=0$, we have obtained a contradiction. Consequently, the matrix $L(j)$ is invertible. 
Proposition 4.5. The sequence $\left\{D\left(\delta_{h}\right)\right\}$ of matrices converges to the matrix $\bar{D}$ with rows given by $\bar{d}_{j}=\bar{\theta}_{j} \mathbb{1}^{\top} L^{-1}(j)$ for $j \in N$.

Proof. We fix $h$ and denote $\delta_{h}$ by $\delta, \theta^{i}\left(\delta_{h}\right)$ by $\theta^{i}$, and $d^{i}\left(\theta^{h}\right)$ by $d^{i}$.

For each $j \in N$ and $i \in N \backslash\{j, n\}$,

$$
\begin{aligned}
d_{j}^{i}(1-\delta) & =\theta_{j}^{i}-\theta_{j}^{n}=\delta \sum_{k=1}^{n} m_{i k} \theta_{j}^{k}-\theta_{j}^{n} \\
& =\delta \sum_{k=1}^{n} m_{i k}\left(\theta_{j}^{k}-\theta_{j}^{n}\right)+\delta \theta_{j}^{n}-\theta_{j}^{n},
\end{aligned}
$$

where we use conditions (3.1) and (3.5) for the second equality, so

$$
d_{j}^{i}=\delta \sum_{k=1}^{n-1} m_{i k} d_{j}^{k}-\theta_{j}^{n} .
$$

We have found that

$$
\theta_{j}^{n}=\delta \sum_{k \notin\{i, n\}} m_{i k} d_{j}^{k}+\left(\delta m_{i i}-1\right) d_{j}^{i}, \quad j \in N, i \in N \backslash\{j, n\} .
$$

Similarly, for $j \neq n$,

$$
\begin{aligned}
d_{j}^{j}(1-\delta) & =\theta_{j}^{j}-\delta \sum_{k=1}^{n} m_{n k} \theta_{j}^{k} \\
& =\theta_{j}^{j}-\delta \sum_{k=1}^{n} m_{n k}\left(\theta_{j}^{k}-\theta_{j}^{n}\right)-\delta \theta_{j}^{n} \\
& =\theta_{j}^{j}-\theta_{j}^{n}-\delta \sum_{k=1}^{n} m_{n k}\left(\theta_{j}^{k}-\theta_{j}^{n}\right)+(1-\delta) \theta_{j}^{n},
\end{aligned}
$$

where we use conditions (3.1) and (3.5) for the first equality, so

$$
d_{j}^{j}=d_{j}^{j}-\delta \sum_{k=1}^{n-1} m_{n k} d_{j}^{k}+\theta_{j}^{n} .
$$

We have found that

$$
\theta_{j}^{n}=\delta \sum_{k=1}^{n-1} m_{n k} d_{j}^{k}, \quad j \in N \backslash\{n\} .
$$

We write (4.1)-(4.2) in vector-matrix notation as

$$
\theta_{j}^{n} \mathbb{1}^{\top}=d_{j}\left(\delta M^{\top}-I\right)_{-n}^{-j}, \quad j \in N .
$$

The matrix $\left(M^{\top}-I\right)_{-n}^{-j}$ is invertible by Proposition 4.4, and so is the matrix $\left(\delta M^{\top}-I\right)_{-n}^{-j}$ for $\delta$ close enough to one. Thus, for every $j \in N$, we can solve the above system for $d_{j}$ as

$$
d_{j}=\theta_{j}^{n} \mathbb{1}^{\top}\left[\left(\delta M^{\top}-I\right)_{-n}^{-j}\right]^{-1} .
$$


As $\delta_{h}$ goes to one, the sequence $\theta_{j}^{n}\left(\delta_{h}\right)$ converges to $\bar{\theta}_{j}$ by Proposition 4.3. Thus the sequence $d_{j}\left(\delta_{h}\right)$ converges to $\bar{\theta}_{j} \mathbb{1}^{\top} L^{-1}(j)$, as desired.

Proposition 4.5 expresses each row $j$ of the matrix $\bar{D}$ as the sum of the rows of the matrix $L^{-1}(j)$ multiplied by the scalar $\bar{\theta}_{j}$.

We show now that each column of the matrix $\bar{D}$ is orthogonal to the normal vector of $V$ at the point $\bar{\theta}$, which is unique by Assumption (A3). This is equivalent to saying that each column of the matrix $\bar{D}$ belongs to the tangent space of $\partial V$ at $\bar{\theta}$. We let $\operatorname{span}(\bar{D})$ denote the column span of the matrix $\bar{D}$.

Proposition 4.6. It holds that $\operatorname{span}(\bar{D})$ is orthogonal to the normal vector of $V$ at $\bar{\theta}$.

Proof. Let $\eta^{i}\left(\delta_{h}\right)$ denote the normal vector of $V$ at the point $\theta^{i}\left(\delta_{h}\right)$. Since $\left\{\theta^{i}\left(\delta_{h}\right)\right\}$ converges to $\bar{\theta}$, the sequence $\left\{\eta^{i}\left(\delta_{h}\right)\right\}$ converges to $\bar{\eta}$, the normal vector to the set $V$ at the point $\bar{\theta}$. By the definition of the normal vector,

$$
\eta^{n}\left(\delta_{h}\right)^{\top}\left(\theta^{i}\left(\delta_{h}\right)-\theta^{n}\left(\delta_{h}\right)\right) \leqslant 0 \quad \text { and } \quad \eta^{i}\left(\delta_{h}\right)^{\top}\left(\theta^{i}\left(\delta_{h}\right)-\theta^{n}\left(\delta_{h}\right)\right) \geqslant 0 .
$$

Dividing by $1-\delta_{h}$ and passing to the limit yields the inequalities $\bar{\eta}^{\top} \bar{d}^{i} \leqslant 0$ and $\bar{\eta}^{\top} \bar{d}^{i} \geqslant 0$, therefore $\bar{\eta}^{\top} \bar{d}^{i}=0$, as desired.

Propositions 4.7 and 4.8 address the dimension of $\operatorname{span}(\bar{D})$. We show that the columns of $\bar{D}$ are linearly independent, thus establishing that $\operatorname{span}(\bar{D})$ equals the tangent space of $\partial V$ at $\bar{\theta}$.

For $j \in N$, let $K_{j}$ be the sum of the rows of the matrix $L^{-1}(j)$, thus

$$
K_{j}=\mathbb{1}^{\top} L^{-1}(j) .
$$

Define $K$ as the $(n \times(n-1))$-matrix with rows $K_{j}$. Proposition 4.7 expresses all rows of $K$ in terms of rows of $L^{-1}(n)$ and the stationary distribution $\mu$ induced by $M$.

Proposition 4.7. Any combination of $n-1$ distinct rows of the matrix $K$ is linearly independent. Furthermore,

$$
K_{j}=\mathbb{1}^{\top} L^{-1}(j)=\mathbb{1}^{\top} L^{-1}(n)-\frac{1}{\mu_{j}}\left(L^{-1}(n)\right)_{j}, \quad j \in N \backslash\{n\} .
$$

Proof. We define $x=\left[M^{\top}-I\right]_{-n}^{n}$. Consider some $j \in N \backslash\{n\}$. It can be verified by a direct computation that

$$
L^{-1}(j)=\left[\begin{array}{c}
\left(L^{-1}(n)\right)_{1}-\frac{\left(L^{-1}(n) x\right)_{1}}{\left(L^{-1}(n) x\right)_{j}}\left(L^{-1}(n)\right)_{j} \\
\vdots \\
\left(L^{-1}(n)\right)_{j-1}-\frac{\left(L^{-1}(n) x\right)_{j-1}}{\left(L^{-1}(n) x\right)_{j}}\left(L^{-1}(n)\right)_{j} \\
\left(L^{-1}(n)\right)_{j+1}-\frac{\left(L^{-1}(n) x\right)_{j+1}}{\left(L^{-1}(n) x\right)_{j}}\left(L^{-1}(n)\right)_{j} \\
\vdots \\
\left(L^{-1}(n)\right)_{n-1}-\frac{\left(L^{-1}(n) x\right)_{n-1}}{\left(L^{-1}(n) x\right)_{j}}\left(L^{-1}(n)\right)_{j} \\
\frac{1}{\left(L^{-1}(n) x\right)_{j}}\left(L^{-1}(n)\right)_{j}
\end{array}\right] .
$$


The formula above is well-known in linear programming and is used to compute the simplex tableau following from a change in basis variables. By definition of the stationary distribution we have

$$
L(n) \mu_{-n}^{\top}+x \mu_{n}=0 .
$$

We multiply this expression by $L^{-1}(n)$ and rearrange to obtain

$$
L^{-1}(n) x=-\frac{1}{\mu_{n}} \mu_{-n}^{\top} .
$$

By substitution, we find that

$$
L^{-1}(j)=\left[\begin{array}{c}
\left(L^{-1}(n)\right)_{1}-\frac{\mu_{1}}{\mu_{j}}\left(L^{-1}(n)\right)_{j} \\
\vdots \\
\left(L^{-1}(n)\right)_{j-1}-\frac{\mu_{j-1}}{\mu_{j}}\left(L^{-1}(n)\right)_{j} \\
\left(L^{-1}(n)\right)_{j+1}-\frac{\mu_{j+1}}{\mu_{j}}\left(L^{-1}(n)\right)_{j} \\
\vdots \\
\left(L^{-1}(n)\right)_{n-1}-\frac{\mu_{n-1}}{\mu_{j}}\left(L^{-1}(n)\right)_{j} \\
-\frac{\mu_{n}}{\mu_{j}}\left(L^{-1}(n)\right)_{j}
\end{array}\right] .
$$

Summing up the rows of $L^{-1}(j)$ we get

$$
\mathbb{1}^{\top} L^{-1}(j)=\sum_{i \in N \backslash\{j, n\}}\left(L^{-1}(n)\right)_{i}+\frac{\mu_{j}-1}{\mu_{j}}\left(L^{-1}(n)\right)_{j}=\mathbb{1}^{\top} L^{-1}(n)-\frac{1}{\mu_{j}}\left(L^{-1}(n)\right)_{j} .
$$

Therefore,

$$
K_{-n}=\left[\mathbb{1}^{\top}-C\right] L^{-1}(n),
$$

where $C$ is the $(n-1)$-diagonal matrix with element $1 / \mu_{i}$ in column $i$.

The matrix $\left[\mathbb{1}^{\top}-C\right]$ is non-singular. Suppose not, then there is $y \neq 0$ such that $\left[\mathbb{1}^{\top}-C\right] y=0$. It follows that $\mathbb{1 1}^{\top} y=C y=\left(y_{1} / \mu_{1}, \ldots, y_{n-1} / \mu_{n-1}\right)^{\top}$, from which it follows in particular that $\mathbb{1}^{\top} y \neq 0$. By pre-multiplying the last equality with the row vector $\left(\mu_{1}, \ldots, \mu_{n-1}\right)$, we find that $\left(1-\mu_{n}\right) \mathbb{1}^{\top} y=\mathbb{1}^{\top} y$, a contradiction since $\mu_{n}>0$. Consequently, the matrix $\left[\mathbb{1}^{\top}-C\right]$ is non-singular.

It follows that $K_{-n}$ is non-singular. Since the labeling of players is arbitrary, we have shown that any combination of $n-1$ distinct rows of the matrix $K$ is linearly independent.

Proposition 4.8. It holds that $\bar{\theta}_{i}>0$ for all $i \in N$. The column span of the matrix $\bar{D}$ has dimension $n-1$.

Proof. We know that $\bar{\theta}_{i} \geqslant 0$ for each $i \in N$. Partition $N$ into the set $N_{0}$ of players $i$ such that $\bar{\theta}_{i}=0$ and the set $N_{+}$of players $i$ such that $\bar{\theta}_{i}>0$.

Suppose that the set $N_{0}$ is non-empty, so that the set $N_{+}$consists of at most $n-1$ elements. We show first that $\bar{\theta} \in \operatorname{span}(\bar{D})$ by constructing a vector $z \in \mathbb{R}^{n-1}$ such that $\bar{D} z=\bar{\theta}$. Since the rows $K_{i}$ of the matrix $K$ corresponding to the elements $i$ of the set $N_{+}$are linearly independent by Proposition 4.7 , there exists a vector $z \in \mathbb{R}^{n-1}$ such that $K_{i} z=1$ for all $i \in N_{+}$. Then $\bar{d}_{i} z=$ 
$\bar{\theta}_{i} K_{i} z=\bar{\theta}_{i}$ for all $i \in N_{+}$. Trivially, $z$ also satisfies the equations $\bar{d}_{i} z=\bar{\theta}_{i} K_{i} z=0=\bar{\theta}_{i}$ for each $i \in N_{0}$.

Let $\bar{\eta}$ be the normal vector to $V$ at the point $\bar{\theta}$. Since $\bar{\eta}$ is orthogonal to $\operatorname{span}(\bar{D})$ by Proposition 4.6, we have $\bar{\eta}^{\top} \bar{\theta}=0$. Since zero is in the interior of $V$ by assumption, the vector $\varepsilon \eta$ is in the set $V$ for $\varepsilon>0$ small enough. But then we have the inequality $\bar{\eta}^{\top}(\varepsilon \bar{\eta}-\bar{\theta})=\varepsilon\left(\bar{\eta}^{\top} \bar{\eta}\right)>0$, contradicting the definition of a normal vector. Consequently, the set $N_{0}$ is empty. We have shown the first part of the proposition.

To prove the second part of the proposition, notice that $\bar{D}$ can be written as the product $T K$, where $T$ is a diagonal matrix with $\bar{\theta}_{i}$ in column $i$. Since $\bar{\theta}_{i}>0$ for each $i \in N$, the matrix $T$ has full rank $n$, and the matrix $K$ has rank $n-1$ by Proposition 4.7. This establishes the second part of the proposition.

We now proof that the gradient of the logarithm of the asymmetric Nash product with weights $\mu$ is orthogonal to the column span of the matrix $\bar{D}$. We observe that for $v \gg 0$,

$$
\ln \rho(v)=\sum_{i \in N} \mu_{i} \ln \left(v_{i}\right)
$$

Proposition 4.9. It holds that $\operatorname{span}(\bar{D})$ is orthogonal to the gradient of the function $\ln \rho$ at the point $\bar{\theta}$.

Proof. The gradient of $\ln \rho$ at $\bar{\theta}$ is the vector $g$ given by $g_{j}=\mu_{j} / \bar{\theta}_{j}, j \in N$. We have the following chain of equations

$$
\begin{aligned}
\sum_{j \in N} g_{j} \bar{d}_{j} & =\sum_{j \in N} \mu_{j} \mathbb{1}^{\top} L^{-1}(j) \\
& =\sum_{j \in N \backslash\{n\}} \mu_{j}\left[\mathbb{1}^{\top} L^{-1}(n)-\frac{1}{\mu_{j}}\left(L^{-1}(n)\right)_{j}\right]+\mu_{n} \mathbb{1}^{\top} L^{-1}(n) \\
& =\mathbb{1}^{\top} L^{-1}(n)-\sum_{j \in N \backslash\{n\}}\left(L^{-1}(n)\right)_{j} \\
& =\mathbb{1}^{\top} L^{-1}(n)-\mathbb{1}^{\top} L^{-1}(n)=0,
\end{aligned}
$$

where the first equality uses the result of Proposition 4.5, and the second one Proposition 4.7. This establishes the proposition.

The proof of Theorem 4.2 is now immediate. The column span of the matrix $\bar{D}$ is orthogonal to the normal vector of $V$ at $\bar{\theta}$ by Proposition 4.6, and at the same time it is orthogonal to the gradient of $\ln \rho$ at $\bar{\theta}$ by Proposition 4.9. Since $\operatorname{span}(\bar{D})$ has dimension $n-1$ (Proposition 4.8), it follows at once that the gradient of the function $\ln \rho$ is proportional to the normal vector to $V$ at $\bar{\theta}$. Hence, the point $\bar{\theta}$ is the maximizer of the function $\ln \rho$ on the strictly positive vectors in the set $V$, as well as the maximizer of the function $\rho$ on the set $V_{+}$.

\section{Conclusion}

In this paper we have provided further non-cooperative support to the asymmetric Nash bargaining solution. We demonstrate that existing results are instances of a much more generally 
valid principle. We consider a bargaining process involving any number of players, an arbitrary irreducible Markov process that determines the selection of the proposer, and any set of feasible payoffs that is bounded, convex, and has a smooth boundary. As long as no agreement is reached, negotiations break down with some fixed probability.

We fully characterize the set of subgame perfect equilibria in stationary strategies. We show that at least one such equilibrium exists and argue that in general there are many such equilibria. We continue by studying the limit of an arbitrary sequence of equilibria when the probability of breakdown goes to zero. We establish that in the limit all players make the same proposal. Moreover, this proposal is the same as the one corresponding to the asymmetric Nash bargaining solution, where the weights in the Nash product are equal to the stationary distribution of the Markov process that determines the selection of the proposer.

One implication is that if players are selected as proposer in some fixed order, then the symmetric Nash bargaining solution is achieved in the limit. This can be seen as a generalization of alternating offer bargaining to more than two players. Another implication is that if players are selected according to time-invariant probabilities, these probabilities are equal to the weights in the Nash product. The symmetric Nash bargaining solution would again result if the timeinvariant probabilities are uniform.

It is noteworthy that the bargaining power of the players is only affected by the stationary distribution of the proposer selection process. The particular shape of the set of feasible payoffs is irrelevant for the weights of the players in the Nash product, as are the particular probabilities by which the proposer in the next period is chosen conditional on the current proposer.

\section{References}

[1] J. Banks, J. Duggan, A bargaining model of collective choice, Amer. Polit. Sci. Rev. 94 (2000) $73-88$.

[2] H. Bester, Bargaining versus price competition in markets with quality unvertainty, Amer. Econ. Rev. 83 (1993) 278-288.

[3] K. Binmore, A. Rubinstein, A. Wolinsky, The Nash bargaining solution in economic modelling, RAND J. Econ. 17 (1986) 176-188.

[4] H. Carlsson, A bargaining model where parties make errors, Econometrica 59 (1991) 1487-1496.

[5] S. Chae, J.A. Yang, An N-person pure bargaining game, J. Econ. Theory 62 (1994) 86-102.

[6] S.J. Cho, J. Duggan, Bargaining foundations of the median Voter theorem, working paper, 2005, pp. 1-32.

[7] P.A. Grout, Investment and wages in the absence of binding contracts: A Nash bargaining approach, Econometrica 52 (1984) 449-460.

[8] J.C. Harsanyi, R. Selten, A General Theory of Equilibrium Selection in Games, MIT Press, Cambridge, 1988.

[9] S. Hart, A. Mas-Colell, Bargaining and value, Econometrica 64 (1996) 357-380.

[10] P.J.J. Herings, A. Predtetchinski, One-dimensional bargaining with Markov recognition probabilities, J. Econ. Theory 145 (1) (2010) 189-215.

[11] K. Hyndman, D. Ray, Coalition formation with binding agreements, Rev. Econ. Stud. 74 (2007) 1125-1147.

[12] E. Kalai, Nonsymmetric Nash solutions and replications of 2-person bargaining, Int. J. Game Theory 6 (1977) $129-133$.

[13] T. Kalandrakis, Equilibria in sequential bargaining games as solutions to systems of equations, Econ. Letters 84 (2004) 407-411.

[14] V. Krishna, R. Serrano, Multilateral bargaining, Rev. Econ. Stud. 63 (1996) 61-80.

[15] K. Kultti, H. Vartiainen, Multilateral non-cooperative bargaining in a general Utility Space, working paper, downloadable from http://personal.inet.fi/tiede/yjs-hv, 2007.

[16] A. Laruelle, F. Valenciano, Noncooperative foundations of bargaining power in committees and the Shapley-Shubik index, Games Econ. Behav. 63 (2008) 341-353.

[17] T. Lensberg, Stability and the Nash solution, J. Econ. Theory 45 (1988) 330-341.

[18] A. Merlo, C. Wilson, A stochastic model of sequential bargaining with complete information, Econometrica 63 (1995) 371-399. 
[19] T. Miyakawa, Non-cooperative foundation of $n$-person asymmetric Nash bargaining solution, Osaka University of Economics Working Paper, No. 2006-02, 2006.

[20] J.F. Nash, The bargaining problem, Econometrica 18 (1950) 155-162.

[21] J.F. Nash, Two-person cooperative games, Econometrica 21 (1953) 128-140.

[22] A. Rubinstein, Perfect equilibrium in a bargaining model, Econometrica 50 (1982) 97-109. 